\title{
Selenium-catalyzed oxidation of alkenes with industrial application potential
}

\author{
Lei $\mathrm{Yu}^{\mathrm{a}, *}$ \\ ${ }^{a}$ School of Chemistry and Chemical Engineering, Yangzhou University, Yangzhou 225002, China
}

*Lei Yu: yulei@yzu.edu.cn

\begin{abstract}
Se-catalyzed reactions have been widely investigated during the last decade. They can be employed in the synthesis of many useful industrial intermediates free of wastes. Because of the green features of these reactions, they are of industrial application potential. This article represents the potential applications of Se-catalyzed oxidation of alkenes in industrial production.

Keywords: Selenium, green chemistry, clean oxidant, fine chemical.

\section{Introduction}

Se catalysis is unfolding during the last decade. ${ }^{1-6}$ Compared with many transition metals, $\mathrm{Se}$ is more eco-friendly because it is a necessary trace element for human beings and can be metabolized in organisms. Se-catalyzed reactions can employ $\mathrm{H}_{2} \mathrm{O}_{2}$ as the clean oxidant and generate no waste other than the water, affording efficient synthetic methods with very high atom-economy. During the last decade, a variety of Se-catalyzed reactions have been reported, including our works on Se-catalyzed green reactions to produce many useful intermediates in fine chemical industry. Herein, we wish to discuss the potential application of the Se-catalyzed oxidation of alkenes in industrial level production.
\end{abstract}

\section{Oxidation of alkenes}

\subsection{Diselenide-catalyzed oxidation of cyclohexene}

In 2013, we reported the oxidation of cyclohexene with $\mathrm{H}_{2} \mathrm{O}_{2}$ catalyzed by $(\mathrm{PhSe})_{2}$ (Scheme 1$)^{7}$ The reaction was very clean and could produce trans-1,2-cyclohexadiol, which was a very important chemical intermediates for producing hydroquinone. The product yield was very high (>96\%), but the reaction required very long time (42 h). In the next year, after a series of catalyst screenings, it was found that $\left[3,5-\left(\mathrm{CF}_{3}\right)_{2} \mathrm{C}_{6} \mathrm{H}_{3} \mathrm{Se}\right]_{2}$ was more efficient catalyst and the reaction time could be reduced to 3-5 hours. $^{8}$

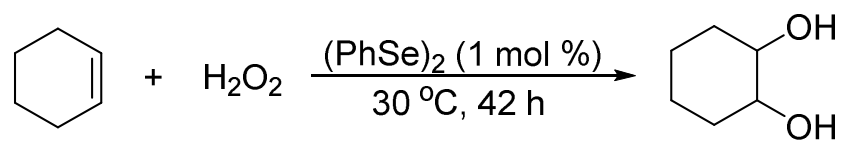

Scheme 1. $(\mathrm{PhSe})_{2}$-catalyzed oxidation of cyclohexene to trans-1,2-cyclohexadiol.

\subsection{Diselenide-catalyzed epoxidation of $\beta$-ionone}

$\beta$-ionone is a cheap fine chemical that can be used as the starting material for preparation spices. In 2016, we found that catalyzed by $\left[3,5-\left(\mathrm{CF}_{3}\right)_{2} \mathrm{C}_{6} \mathrm{H}_{3} \mathrm{Se}\right]_{2}$, the epoxidation of $\beta$-ionone could occur to produce (E)-4-(2,2,6-trimethyl-7-oxabicyclo[4.1.0]heptan-1-yl)but-3 -en-2-one, which was the key intermediate for the synthesis of plant hormone abscisic acid (Scheme 2). Interestingly, when using dibenzyl diselenide, the selectivity of the reaction could be switched to the Baeyer-Villiger reaction of the carbonyl site. ${ }^{9}$

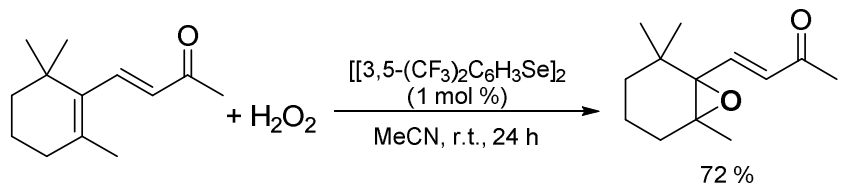

Scheme 2. [3,5- $\left.\left(\mathrm{CF}_{3}\right)_{2} \mathrm{C}_{6} \mathrm{H}_{3} \mathrm{Se}\right]_{2}$-catalyzed epoxidation of $\beta$-ionone.

\subsection{Diselenide-catalyzed oxidative ring expansion of methylenecyclopropane}


Methylenecyclopropanes are a kind of building blocks in organic synthesis. The oxidative ring-expansion of methylenecyclopropanes can produce 2-substituted cyclobutanones, which are useful intermediates in medicine synthesis (Scheme 3). ${ }^{10-12}$ In 2016, we found that, by using diselenide catalysts, the reaction could occur with $\mathrm{H}_{2} \mathrm{O}_{2}$ oxidant and the method generated no waste other than water. ${ }^{10}$

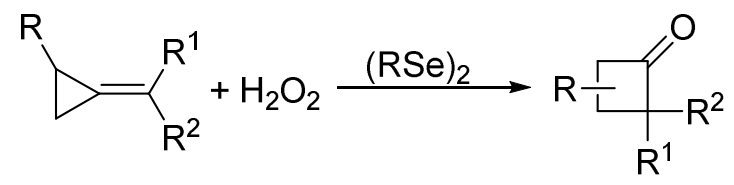

Scheme 3. (RSe) ${ }_{2}$-catalyzed oxidative ring expansion of methylenecyclopropane.

\subsection{Development of the heterogeneous Se catalyst}

In order to develop the recyclable Se catalyst so that the catalyst cost could be reduced, in 2016, we synthesized the polystyrene-supported $\mathrm{Se}$ catalyst (Scheme 4). ${ }^{13}$ The reaction of polystyrene with $n$-BuLi first led to the Li salt, which then reacted with $(\mathrm{MeSe})_{2}$ to produced the supported -SeMe catalyst. The material could react with bromine and was further oxidized by $\mathrm{H}_{2} \mathrm{O}_{2}$ to produce the polystyrene supported selenic acid, which found to be a good catalyst for the oxidation of cyclohexene to trans-1,2-cyclohexadiol.
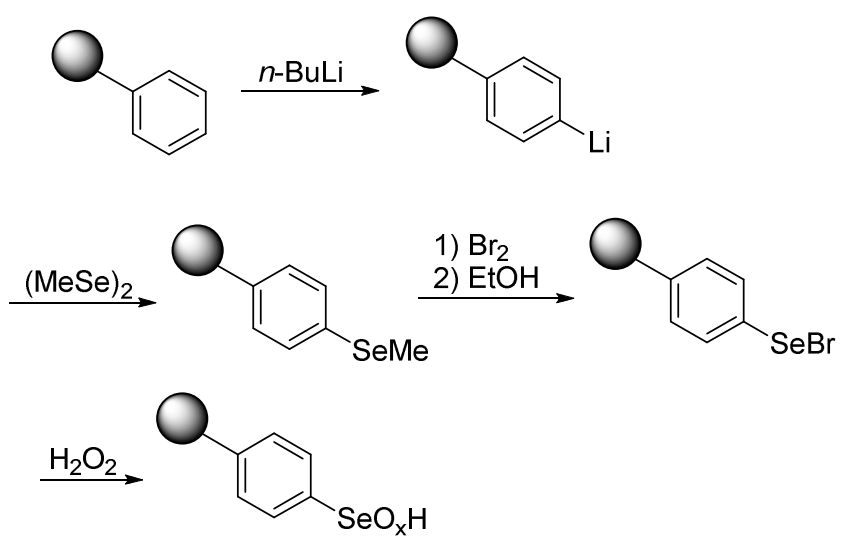

Scheme 4. Preparation of the polystyrene-supported Se catalyst.

Although the polystyrene-supported selenic acid could be easily recycled and reused for several times without deactivation, its preparation cost was high due to the tedious fabrication procedures and the use of harsh and expensive reagents. In order to reduce the cost of the catalyst, in 2018, we developed the Se/C catalyst from carbohydrates: ${ }^{14}$ The reduction of Se by $\mathrm{NaBH}_{4}$ could generate the NaHSe, which was a strong selenization reagent and could react with glucose to prepared the Se-substituted glucose. Calcining the Se-substituted glucose led to the $\mathrm{Se} / \mathrm{C}$ materials, which could catalyze the epoxidation of $\beta$-ionone to produce the useful chemical (E)-4-(2,2,6-trimethyl-7-oxabicyclo[4.1.0]heptan-1-yl)but-3 -en-2-one and the turnover number of the catalyst could reach $2 \times 10^{5}$.

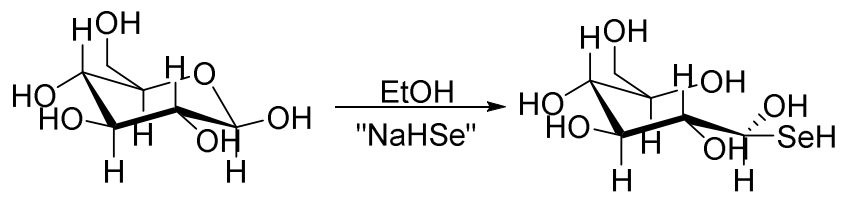

$$
\stackrel{500^{\circ} \mathrm{C}}{\longrightarrow}
$$

Scheme 5. Preparation of the Se/C catalyst.

\section{Conclusions}

In conclusion, as shown above, the Se-catalyzed oxidation of alkenes could produce useful chemicals, such as trans-1,2-cyclohexadiol, 2-substituted cyclobutanones, (E)-4-(2,2,6-trimethyl-7-oxabicyclo[4.1.0]heptan-1-yl)but-3 -en-2-one, etc. The heterogeneous Se catalysts were also developed and applied in the related reactions, which were of very good application potential from the industrial viewpoint.

\section{Acknowledgment}

We thank National Key Research and Development Program of China (2018YFD0200100), National Natural Science Foundation of China (21202141), Natural Science Foundation of Jiangsu Province (BK20181449), Jiangsu Provincial Six Talent Peaks Project (XCL-090), and Priority Academic Program Development of Jiangsu Higher Education Institutions (PAPD) for financial support.

\section{References}

(1) D. M. Freudendahl, S. Santoro, S. A. Shahzad, C. Santi and T. Wirth, "Green chemistry with selenium reagents: development of efficient catalytic reactions", Angew. Chem. Int. Ed., 2009, 48, 8409.

(2) C. Santi, S. Santoro and B. Battistelli, "Organoselenium compounds as catalysts in nature and laboratory”, Curr. Org. Chem., 2010, 14, 2442.

(3) S. Santoro, J. B. Azeredo, V. Nascimento, L. Sancineto, 
A. L. Braga and C. Santi, "The green side of the moon: ecofriendly aspects of organoselenium chemistry", RSC Adv., 2014, 4, 31521.

(4) J. Młochowski and H. Wójtowicz-Młochowska, "Developments in synthetic application of selenium(IV) oxide and organoselenium compounds as oxygen donors and oxygen-transfer agents", Molecules, 2015, 20, 10205.

(5) A. Breder and S. Ortgies, "Recent developments in sulfur- and selenium-catalyzed oxidative and isohypsic functionalization reactions of alkenes", Tetrahedron Lett., 2015, 56, 2843.

(6) R. Guo, L. Liao and X. Zhao, "Electrophilic selenium catalysis with electrophilic N-F reagents as the oxidants", Molecules, 2017, 22, 835.

(7) L. Yu, J. Wang, T. Chen, K. Ding and Y. Pan, "Access to cyclohexane-1,2-diol through the diphenyldiselenide catalyzed oxidation of cyclohexene by hydrogen peroxide", Chin. J. Org. Chem., 2013, 33, 1096.

(8) L. Yu, J. Wang, T. Chen, Y. Wang and Q. Xu, "Recyclable 1,2-bis[3,5-bis(trifluoromethyl)phenyl] diselane-catalyzed oxidation of cyclohexene with H2O2: a practical access to trans-1,2-cyclohexanediol”, Appl. Organomet. Chem., 2014, 28, 652.

(9) L. Yu, Z. Bai, X. Zhang, X. Zhang, Y. Ding and Q. Xu, "Organoselenium-catalyzed selectivity-switchable oxidation of $\beta$-ionone", Catal. Sci. Technol., 2016, 6, 1804.

(10) W.-L. Chen, X. Huang, H.-W. Zhou and L.-J. Ren, "Ceric(IV)-mediated reaction of methylene -cyclopropanes in organic Synthesis: A facile access to dihydrofurans and cyclobutanones", Synthesis, 2006, 609.

(11) V. Nair, T. D. Suja and K. Mohanan, "A convenient synthesis of 2,2-diarylcyclobutanones by cerium(IV) ammonium nitrate (CAN) mediated oxidation of methylenecyclopropanes (MCPs)", Synthesis, 2006, 2531.

(12)L.-X. Shao and M. Shi, "Ring-expansion of MCPs in the presence of DIAD or DEAD and Lewis acids", Eur. J. Org. Chem., 2004, 426.

(13) Y. Wang, L. Yu, B. Zhu and L. Yu, "Design and preparation of polymer resin-supported organoselenium catalyst with industrial potential", $J$. Mater. Chem. A, 2016, 4, 10828.

(14) Y.-F. Yang, X. Fan, H.-E. Cao, S.-N. Chu, X. Zhang, Q. $\mathrm{Xu}$ and $\mathrm{L}$. $\mathrm{Yu}$, "Fabrication of $\mathrm{Se} / \mathrm{C}$ by using carbohydrate as the biomass starting materials: an efficient catalyst for the regio-specific epoxidation of $\beta$-ionone with ultrahigh turnover numbers", Catal. Sci. Technol., 2018, 8, 5017. 